\title{
Mechanisms of ultrasonic modulation of multiply scattered coherent light: a Monte Carlo model
}

\author{
Lihong V. Wang \\ Optical Imaging Laboratory, Biomedical Engineering Program, Texas A\&M University, 3120 TAMU, College Station, Texas 77843-3120
}

Received February 20, 2001

\begin{abstract}
A Monte Carlo model of the ultrasonic modulation of multiply scattered coherent light in scattering media is provided. The model is based on two mechanisms: the ultrasonic modulation of the index of refraction, which causes a modulation of the optical path lengths between consecutive scattering events, and the ultrasonic modulation of the displacements of scatterers, which causes a modulation of optical path lengths with each scattering event. Multiply scattered light accumulates modulated optical path lengths along its path. Consequently, the intensity of the speckles that are formed by the multiply scattered light is modulated. The contribution from the index of refraction is comparable with the contribution from displacement when the acoustic-wave vector is less than a critical fraction of the transport mean free path and becomes increasingly greater than the contribution from displacement beyond this critical point. This Monte Carlo model agrees well with an independent analytical model for isotropically scattering media. Both mechanisms are coherent phenomena, requiring the use of a coherent light source. (C) 2001 Optical Society of America
\end{abstract}

OCIS codes: $290.7050,290.4210,110.7170,030.1670,030.6600$.

Ultrasound-modulated optical tomography is a new area of research for the imaging of scattering media, such as biological tissues. In this technology an ultrasonic wave is focused into a scattering medium to modulate light inside the medium. The advantage of this technology is its combination of optical contrast and ultrasonic resolution. Marks et al. investigated the modulation of light in homogeneous scattering media with pulsed ultrasound. ${ }^{1}$ Wang et al. developed ultrasound-modulated optical tomography in scattering media. ${ }^{2}$ Kempe et al. showed experimentally the transition of ultrasonic modulation from the ballistic to the scattered regime. ${ }^{3}$ Wang and Ku developed a frequency-swept technique with which to obtain scalable imaging resolution along the acoustic axis. ${ }^{4}$ Leveque et al. employed parallel detection of multiple speckles to improve signal-to-noise ratio, which was demonstrated with one-dimensional images of biological tissue. $^{5}$ Yao and Wang further demonstrated this parallel-detection scheme with two-dimensional images of biological tissue. ${ }^{6}$ In addition, by combining the parallel-detection and the frequency-swept techniques, Yao et al. obtained two-dimensional images with resolution along the acoustic axis. ${ }^{7}$ Lev et al. developed a reflection configuration. ${ }^{8}$

Three possible mechanisms have been identified for the ultrasonic modulation of light in scattering media; which mechanism is dominant has, however, remained a mystery. The first mechanism is based on ultrasound-induced variations of the optical properties of the media. As an ultrasonic wave propagates in a scattering medium, the medium is compressed and rarified depending on location and time. Variations of density cause the optical properties of the medium to vary. Accordingly, the detected intensity of light varies with the ultrasonic wave. This mechanism, recently modeled by Mahan et al., ${ }^{9}$ does not require the use of a coherent light source; however, it is too weak to have been experimentally observed.

The second mechanism is based on variations of the optical phase in response to ultrasound-induced displacements of scatterers. The displacements of scatterers, assumed to follow ultrasonic amplitudes, modulate the physical path lengths of light traversing the ultrasonic field. Multiply scattered light accumulates modulated physical path lengths along its path. Consequently, the intensity of the speckles formed by the multiply scattered light fluctuates with the ultrasonic wave. A theory by Leutz and Maret ${ }^{10}$ modeled this mechanism but is valid only when the scattering mean free path is much greater than the acoustic wavelength.

The third mechanism is based on variations of the optical phase in response to ultrasonic modulation of the index of refraction, which vary when the optical phase between two consecutive scattering events is modulated. As in the second mechanism, multiply scattered light accumulates modulated phases along its path, and the modulated phases cause the intensity of the speckles formed by the multiply scattered light to vary with the ultrasonic wave. This mechanism has apparently never been modeled.

Both the second and the third mechanisms require the use of coherent light. In this Letter I present a Monte Carlo model based, for what is to my knowledge the first time, on both of the mechanisms for the ultrasonic modulation of coherent light in scattering media. The Monte Carlo model is compared with an analytical model for isotropically scattering media based on diffusion theory. The Monte Carlo model is not subject to the limitations of diffusion theory and is studied here for anisotropically scattering media. The applicability of the analytical model to anisotropically scattering media is numerically validated.

A plane ultrasonic wave is assumed to insonify uniformly a homogeneous scattering medium. The autocorrelation function of the electric field, $E(t)$, of the scattered light can be expressed as

$$
G_{1}(\tau)=\int_{0}^{\infty} p(s)\left\langle E_{s}(t) E_{s}{ }^{*}(t+\tau)\right\rangle \mathrm{d} s,
$$


where the averaging is over time $t, E_{s}$ is the unit electric field of the scattered light to path length $s$, and $p(s)$ is the probability density function of $s$. The contributions to the autocorrelation function from Brownian motion and from the ultrasonic field are independent and can be treated separately. For conciseness, only the ultrasonic contribution is considered here.

The ultrasonic contribution to the autocorrelation function from a path of length $s$ is

$$
\left\langle E_{s}(t) E_{s}^{*}(t+\tau)\right\rangle=\langle\exp [-i \Delta \phi(t, \tau)]\rangle,
$$

where $\Delta \phi(t, \tau)=\sum_{j}^{N+1} \Delta \phi_{n j}(t, \tau)+\sum_{j}^{N} \Delta \phi_{d j}(t, \tau)$, $\Delta \phi_{n j}(t, \tau)=\phi_{n j}(t+\tau)-\phi_{n j}(t), \phi_{n j}$ is the phase variation induced by the modulated index of refraction along the $j$ th free path, $\Delta \phi_{d j}(t, \tau)=\phi_{d j}(t+\tau)-\phi_{d j}(t)$, $\phi_{d j}$ is the phase variation induced by the modulated displacement of the $j$ th scattering site following the $j$ th free path, and $N$ is the number of scattering events along the full path. There is an additional free path to the detector after the last scattering event.

On the one hand, the phase variation from the $j$ th free path is

$$
\phi_{n j}(t)=\int_{0}^{l_{j}} k_{0} \Delta n\left(\mathbf{r}_{j-1}, s_{j}, \theta_{j}, t\right) \mathrm{d} s_{j},
$$

where $l_{j}$ is the length of the $j$ th free path, $k_{0}$ is the optical wave vector in vacuo, $\Delta n$ is the modulated index of refraction, $\mathbf{r}_{j}$ is the location of the $j$ th scatterer, $s_{j}$ is the distance along the $j$ th free path, and $\theta_{j}$ is the angle between the optical wave vector of the $j$ th free path and the acoustic-wave vector $\mathbf{k}_{a}$.

The modulated index of refraction is

$$
\begin{aligned}
\Delta n\left(\mathbf{r}_{j-1}, s_{j}, \theta, t\right)= & n_{0} \eta k_{a} A \sin \left(\mathbf{k}_{a} \cdot \mathbf{r}_{j-1}\right. \\
& \left.+k_{a} s_{j} \cos \theta_{j}-\omega_{a} t\right),
\end{aligned}
$$

where $n_{0}$ is the background index of refraction, $k_{a}$ is the amplitude of $\mathbf{k}_{a}, A$ is the acoustic amplitude, $\omega_{a}$ is the acoustic angular frequency, and $\eta$ is related to the adiabatic piezo-optical coefficient of the material, $\partial n / \partial p$, the density $\rho$, and the acoustic velocity $\nu_{a}: \quad \eta=(\partial n / \partial p) \rho \nu_{a}^{2}$.

Completing the integration in Eq. (3), one obtains

$$
\begin{aligned}
\phi_{n j}(t)= & 2 n_{0} k_{0} \eta A \sin \left(\mathbf{k}_{a} \cdot \mathbf{r}_{j-1}+k_{a} l_{j} \cos \theta_{j} / 2-\omega_{a} t\right) \\
& \times \sin \left(k_{a} l_{j} \cos \theta_{j} / 2\right) / \cos \theta_{j} .
\end{aligned}
$$

On the other hand, the phase variation from the $j$ th scattering event is

$$
\phi_{d j}(t)=-n_{0} k_{0}\left(\hat{\mathbf{k}}_{j+1}-\hat{\mathbf{k}}_{j}\right) \cdot \mathbf{A} \sin \left(\mathbf{k}_{a} \cdot \mathbf{r}_{j}-\omega_{a} t\right),
$$

where $\hat{\mathbf{k}}_{j}$ is the unit optical wave vector for the $j$ th free path and $\mathbf{A}$ is the acoustic-amplitude vector.

A public-domain software package for incoherent light transport in scattering media based on a Monte Carlo technique ${ }^{11,12}$ was modified to model ultrasonic modulation of coherent light in scattering media. In this modified model a coherent optical beam from an extended plane source is incident upon a slab of thickness $L$, and the transmitted light is detected from a point. A direct solution to this problem by use of the Monte Carlo technique would be computationally inefficient. Exploiting the principle of reciprocity makes it possible to overcome this problem. In the reciprocal problem, a point source illuminates the slab, and a plane detector detects the transmitted light.

The new Monte Carlo model is briefly described as follows: (1) Launch a photon packet from the point of incidence into the scattering medium. (2) Determine the step size by $l_{j}=-\ln (\xi) / \mu_{s}$, where $\xi$ is a random number evenly distributed between 0 and 1 and $\mu_{s}$ is the scattering coefficient. (3) If the step size fits in the scattering medium, move the photon packet to the new location. Accumulate $\Delta \phi_{n j}(t, \tau)$ based on Eq. (5), where both $t$ and $\tau$ vary from 0 to the acoustic period, $T_{a}$, with a given number of divisions. (4) Scatter the photon packet in a new direction based on the scattering phase function (form factor): a Henyey-Greenstein function for the polar angle, and a uniform function for the azimuth angle. Accumulate $\Delta \phi_{d j}(t, \tau)$ based on Eq. (6) similarly to step 3. Then, go back to step 2 to continue tracing the photon packet. (5) If the step size crosses the incident surface, the photon packet is diffusely reflected and is terminated. (6) If the step size crosses the transmitting surface, the photon packet is transmitted and is terminated. The contribution of this photon packet to the autocorrelation function is recorded by use of Eq. (2). (7) Repeat steps (1)-(6) and sum the autocorrelation functions from all the photon packets to obtain the overall autocorrelation function. (8) The light intensity at frequency $n \omega_{a}$ is calculated based on the Wiener-Khinchin theorem: $I_{n}=\int_{0}^{T_{a}} \cos \left(n \omega_{a} \tau\right) G_{1}(\tau) \mathrm{d} \tau / T_{a}$. The modulation depth, $M=I_{1} / I_{0}$, is then calculated.

Figure 1(a) shows the modulation depth contributed by the index of refraction alone, $M_{n}$; the modulation depth contributed by displacement alone, $M_{d}$; and the modulation depth contributed by both, $M_{\text {sum }}$. Figure 1(b) is a plot of the ratio between the two types of contribution: $M_{n} / M_{d}$. The symbols, from the Monte Carlo model, agree with the lines, from an independent analytical model based on diffusion theory that is applicable for isotropically scattering media (anisotropy $g=0$ ). ${ }^{13} \quad M_{n}$ increases with $k_{a}$, whereas $M_{d}$ does not vary with $k_{a}$. The former is because the average phase accumulation from the free paths increases with $k_{a}$, and the latter is because the average phase accumulation from the scattering events does not depend on $k_{a}$. The ratio $M_{n} / M_{d}$ shows that $M_{n}$ is comparable with $M_{d}$ when $k_{a}$ is less than a critical value-found to be $0.559 \mu_{s}$ from the analytical model-but $M_{n}$ becomes greater thereafter. Experimental verification of this theoretical observation can be explored with scatterers of various weights. ${ }^{14}$

Figure 2 shows $M_{n}$ and $M_{d}$ as a function of the scattering anisotropy, $g$. The modulation depths are found to be insensitive to $g$ if a constant transport scattering coefficient, $\mu_{s}(1-g)$, is maintained. A 

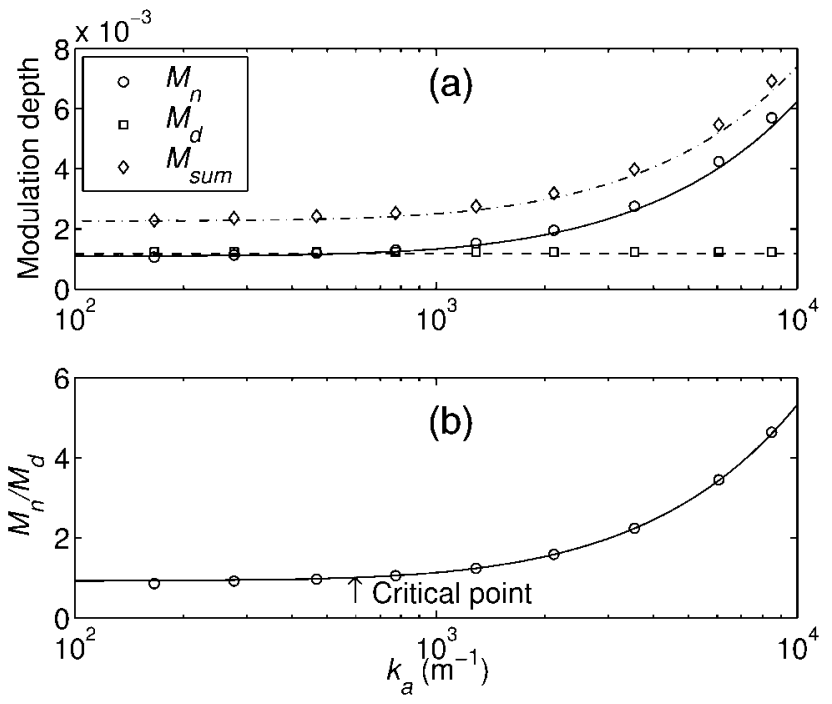

Fig. 1. (a) $M_{n}, M_{d}$, and $M_{\text {sum }}$ and (b) $M_{n} / M_{d}$ versus $k_{a}$. The parameters are $\partial n / \partial p=1.466 \times 10^{-10} \mathrm{~m}^{2} / \mathrm{N}$, $\rho=1000 \mathrm{~kg} / \mathrm{m}^{3}, \nu_{a}=1480 \mathrm{~m} / \mathrm{s}, A=0.1 \mathrm{~nm}, n_{0}=1.33$, $\lambda_{0}=500 \mathrm{~nm}, \mu_{s}=10 \mathrm{~cm}^{-1}, g=0$, and $L=5 \mathrm{~cm}$.

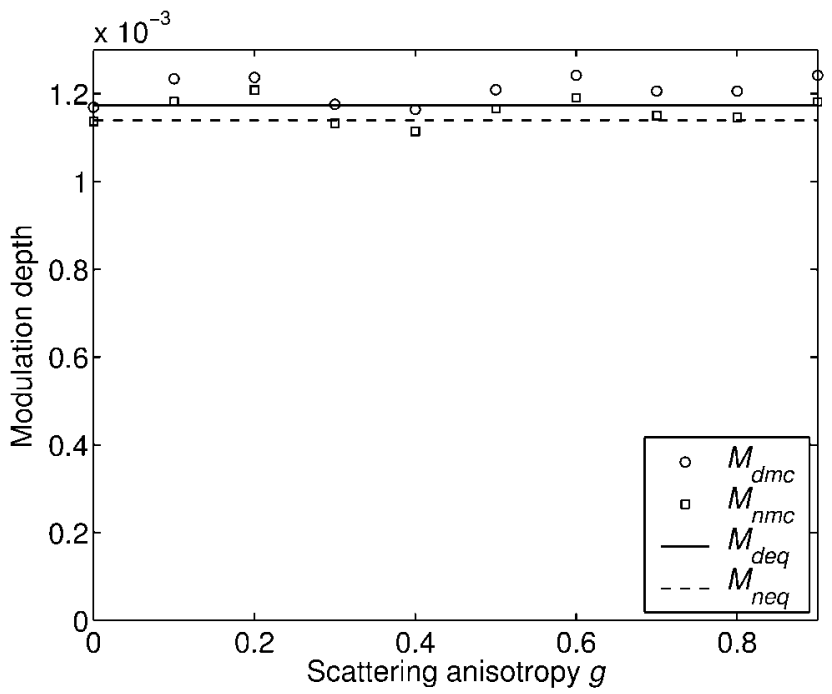

Fig. 2. $M_{n}$ and $M_{d}$ versus $g$ while $\mu_{s}(1-g)$ is kept constant. The subscript $m c$ indicates the results from the Monte Carlo model; and the subscript eq represents the results from the analytical model, where the modulation depths for $g>0$ are assumed to be equal to those for $g=0$. The parameters in Fig. 1 are used, except that $T_{a}=10 \mu \mathrm{s}$ and $\mu_{s}(1-g)=10 \mathrm{~cm}^{-1}$.

direct analytical solution for anisotropically scattering media is not readily available; however, the analytical solution for the corresponding isotropically scattering medium can be used to calculate the modulation depths. Consequently, the observation about the relative contributions to the modulation depth of the two mechanisms in an isotropically scattering medium [Fig. 1(b)] can be extended to anisotropically scattering media if $\mu_{s}$ of the isotropically scattering medium is replaced with $\mu_{s}(1-g)$ of the anisotropically scattering media. Of course, this conclusion is applicable only in the diffusion regime because the analytical model is based on diffusion theory, although the Monte Carlo model can be used in either the diffusion or the nondiffusion regime. The Monte Carlo model is flexible and can be adapted to simulate more-complex configurations such as localized ultrasonic waves and inhomogeneous scattering media.

In summary, a Monte Carlo model of the ultrasonic modulation of multiply scattered coherent light in scattering media has been provided. The model is based on two coherent mechanisms: ultrasonic modulation of the index of refraction, which causes a modulation of the optical path lengths between consecutive scattering events, and ultrasonic modulation of the displacements of scatterers, which causes a modulation of optical path lengths with each scattering event. Multiply scattered light accumulates modulated optical path lengths along its path. Consequently, the intensity of the speckles that are formed by the multiply scattered light is modulated. The contribution from the index of refraction is comparable with the contribution from displacement when the acoustic-wave vector is less than a critical fraction of the transport mean free path and becomes increasingly greater than the contribution from displacement beyond this critical point. This Monte Carlo model agrees well with an independent diffusion-based analytical model for isotropically scattering media. The analytical model is further shown to be valid for anisotropically scattering media. This work clarifies the mystery about the mechanisms of ultrasonic modulation of light in scattering media.

This project was sponsored in part by National Science Foundation grant BES-9734491, Texas Higher Education Coordinating Board grant 000512-0123-1999, and National Institutes of Health grants R29 CA68562 and R01 CA71980. The author's e-mail address is lwang@tamu.edu.

\section{References}

1. F. A. Marks, H. W. Tomlinson, and G. W. Brooksby, Proc. SPIE 1888, 500 (1993).

2. L.-H. Wang, S. L. Jacques, and X. Zhao, Opt. Lett. 20, 629 (1995).

3. M. Kempe, M. Larionov, D. Zaslavsky, and A. Z. Genack, J. Opt. Soc. Am. 14, 1151 (1997).

4. L.-H. V. Wang and G. Ku, Opt. Lett. 23, 975 (1998).

5. S. Leveque, A. C. Boccara, M. Lebec, and $H$. Saint-Jalmes, Opt. Lett. 24, 181 (1999).

6. G. Yao and L.-H. V. Wang, Appl. Opt. 39, 659 (2000).

7. G. Yao, S.-L. Jiao, and L.-H. V. Wang, Opt. Lett. 25, 734 (2000).

8. A. Lev, Z. Kotler, and B. G. Sfez, Opt. Lett. 25, 378 (2000).

9. G. D. Mahan, W. E. Engler, J. J. Tiemann, and E. Uzgiris, Proc. Natl. Acad. Sci. USA 95, 14015 (1998).

10. W. Leutz and G. Maret, Physica B 204, 14 (1995).

11. L.-H. Wang, S. L. Jacques, and L.-Q. Zheng, Comput. Methods Programs Biomed. 47, 131 (1995).

12. Software available at http://oilab.tamu.edu./mc.html.

13. L.-H. Wang, Proc. SPIE 4256, 208 (2001).

14. C. A. DiMarzio, R. J. Gaudette, and T. J. Gaudette, Proc. SPIE 3597, 376 (1999). 